\title{
La evaluación de la competencia oral en segundas lenguas. El caso del inglés en el mundo profesional
}

\author{
$\mathrm{M}^{\mathrm{a}}$ Lourdes POMPOSO YANES \\ Departamento de Inglés. Facultad de Educación \\ Universidad Camilo José Cela, Madrid \\ lpomposo@ucjc.edu
}

Recibido: 21 de marzo de 2016

Aceptado: 5 de julio de 2016

\section{RESUMEN}

La evaluación de la competencia oral es una de las más subjetivas en la enseñanza de una segunda lengua y a veces la que más conflictos origina en cuanto la evaluación justa e injusta dependiendo de los objetivos de dicha evaluación. Además del problema de la subjetividad hay que añadir la complejidad del consumo de tiempo que requiere si se quiere realizar de forma individual, la preparación de la actividad, la rúbrica específica que se quiere utilizar y las exigencias del proceso o protocolo que se debería de seguir para una correcta evaluación.

En este documento realizaremos un planteamiento teórico de la evaluación de la competencia oral en segundas lenguas, el proceso de evaluación y la importancia de las escalas de valoración. Veremos las que son utilizadas por las instituciones más reconocidas en la enseñanza y evaluación del inglés además de sus descriptores, poniendo ejemplos de dicha evaluación en el mundo laboral y además propondremos una rúbrica para evaluar de forma holística que puede ser utilizada en un proceso de selección de candidatos a un trabajo.

Palabras clave: Evaluación oral, procesos de evaluación, segundas lenguas, inglés empresarial

The evaluation of oral competence in second languages. The case of English in the professional environment

\begin{abstract}
The evaluation of the oral competence is one of the most subjective ones in second language teaching and sometimes the most conflictive one related to the fair or unfair testing depending on the aims of that evaluation. Apart from the subjectivity problem we have to add the difficulty of the required time consuming fact, mainly if that evaluation is carried out in an individual way, the activity preparation, the right rubric and all the requirements that a good evaluation process or protocol implies.

In this document we will display a theoretical framework of evaluation of oral competence in second language acquisition, the evaluation process, the importance of rating scales and their descriptors studying the most commonly used in well-known institutions and we
\end{abstract}


present a holistic rate scale to be presumably used in the process of candidates selection in a job interview.

Key words: Oral evaluation, evaluation processes, second languages, business English

L'évaluation de la compétence orale dans les secondes langues. Le cas de

\section{RÉSUMÉ}

l'anglais dans le monde professionnel

L'évaluation de la compétence orale dans l'enseignement d'une seconde langue est l'une des plus subjectives qui soient, et parfois celle qui suscite le plus de conflits, concernant l'évaluation juste et injuste, selon les objectifs poursuivis par cette évaluation. Outre ce problème de la subjectivité, il faut ajouter la complexité liée au temps qu'elle consomme si l'on veut la réaliser de façon individuelle, ainsi que la préparation de l'activité, la rubrique spécifique dont on veut se servir et les exigences du processus ou du protocole à suivre pour une correcte évaluation.

Dans le présent document nous poserons une approche théorique de l'évaluation de la compétence orale dans les secondes langues, ainsi que du processus d'évaluation et de l'importance des échelles de valoration. Nous verrons celles qui sont utilisées par les institutions les plus réputées dans l'enseignement et l'évaluation de l'Anglais, avec leurs descripteurs, et nous donnerons des exemples de cette évaluation dans le monde du travail et de plus nous poposerons une échelle de valeur holistique qui pourra être utilisée dans la s'election des candidats à l'offre d'emploi.

Mots-clés: Évaluation orale, processus d'évaluation, secondes langues, anglais des affaires

SUMARIO: 1 . Características de la competencia oral. Dificultad de la evaluación de una segunda lengua; 1.1. El inglés en el mundo empresarial; 1.2. El proceso de evaluación de la competencia oral; 1.3. La evaluación de la competencia oral en la producción individual y la interacción; 2. Las escalas de valoración de la competencia oral; 2.1. Tipos de escalas de valoración; 2.2. Propuesta de escala holística; 3. Conclusiones; 4. Bibliografía.

\section{CARACTERÍSTICAS DE LA COMPETENCIA ORAL. DIFICULTAD DE LA EVALUACIÓN DE UNA SEGUNDA LENGUA}

Evaluar la expresión oral en una segunda lengua suele resultar una tarea difícil y problemática, ya que no es tan objetivable como la escrita. Tal y como observan Alderson, Clapham y Wall $(1998,89)$ :

Es una prueba subjetiva y la corrección misma puede que no resulte fiable. Esto puede ser debido a factores como la variación en las distintas formas en que se llevó a cabo la evaluación, la ambigüedad de los criterios de evaluación, la aplicación de distintos correctores o la inconsistencia por parte de algunos.

Esa ambigüedad o subjetividad suele ser la clave de la dificultad de la evaluación oral de una segunda lengua, algo que no ocurre con la evaluación de la lengua escrita, en la cual es más sencillo identificar y cuantificar los factores que componen su calidad. 
En la evaluación de la competencia oral, intervienen numerosos factores comunicativos, ya que la comunicación oral implica la negociación de significados entre dos o más personas que se encuentran en un contexto determinado (O'Malley y Pierce, 1996). Esos factores no son sólo lingüísticos, tales como la pronunciación, la gramática, la fluidez o la comprensión, sino pragmáticos, que hacen que el mensaje oral sea efectivo desde el punto de vista comunicativo, especialmente en contextos profesionales. Tal como indican Canale y Swain (1980), en la competencia comunicativa se combinan ciertas reglas de tipo discursivo con otras de tipo sociocultural que son totalmente necesarias para desambiguar determinados enunciados en los que no queda clara la intención del hablante. Este conjunto de reglas tienen una estrecha relación con el concepto de adecuación de los enunciados al contexto en el que el hablante se encuentra (Hymes, 1972).

El concepto de competencia comunicativa se ha ido ampliando a lo largo de los años y en consecuencia a las distintas situaciones profesionales y no profesionales en las que la lengua se desarrolla. De hecho Bachman (1990) considera que la comunicación no debe ser descrita en el uso individual como una competencia, sino como una habilidad:

Communicative language ability can be described as consisting of both knowledge, or competence, and the capacity form implementing or executing that competence in appropriate, contextualized communicative language use (Bachman, 1990, 84).

El modelo de Bachmnan está reelaborado desde las ideas iniciales expuestas en Bachman y Palmer (1982), donde se expone que la destreza comunicativa es un compendio de competencia gramatical, competencia pragmática y competencia sociolingüística. De ahí que diseñaran un paradigma comunicativo basado en la competencia organizativa, referida al control de la gramática, en la competencia pragmática, referida a la relación entre el contexto y el hablante. Podemos deducir, entonces, que el uso de la lengua está relacionado con las destrezas cognitivas individuales de cada persona (McNamara, 1996), por lo que los resultados de la producción oral ya sean de un hablante nativo o un hablante no nativo pueden diferir según su experiencia, cultura o competencias. El objetivo de cualquier aprendiz de lenguas es el de parecerse a un hablante nativo ideal y en este esfuerzo intervienen factores de orden cognitivo y sociocultural. De hecho, las investigaciones llevadas a cabo por Weir (1998), que realizó pruebas orales a hablantes nativos y no nativos, demostraron que los hablantes nativos no conseguían al 100\% una comunicación efectiva. Widdowson $(1978,59)$ afirma que la comunicación efectiva es la que se produce en un entorno real y cotidiano en el que se produce un diálogo comprensible y real entre un emisor y un receptor. En ese diálogo también intervienen elementos paralingüísticos tales como gestos, tartamudeos, interrupciones, risas, muecas o lo que Canale (1995) llamó competencia estratégica. Esta estrategia viene a suplir las deficiencias que puedan 
darse debido a errores en el desarrollo de la puesta en práctica de la lengua o relacionada con la competencia de los hablantes. Ésta está relacionada con la competencia sociolingüística y se refiere a la estrategia compensatoria de, por ejemplo, parafrasear un significado o definición cuando no se sabe una palabra determinada y no se quiere interrumpir la comunicación.

Los parámetros que intervienen en la interpretación de un enunciado oral son numerosos y concomitantes: a los elementos morfosintácticos y semánticos, hay que agregarles especialmente la prosodia, como la entonación, las variaciones de cadencia o las pausas. (García-Debenc, 2010, 105). Según Widdowson (1978), cualquier comunicación oral hay que medirla desde dos puntos de vista: el formal y el funcional. El uso formal de la lengua, lo que él llama usage, se refiere a la habilidad de producir frases gramaticalmente correctas dando importancia a la prescripción a la hora de hablar. El punto de vista funcional se refiere a lo que él llama use. Es la habilidad de utilizar el conocimiento de tal manera que se produzca una comunicación efectiva. Es, en resumen, cómo se utiliza la lengua.

Weir (2005) añade que para ser capaces de comunicarse en una segunda lengua, es necesario enlazar las estructuras de la lengua sintáctica y fonológicamente y tener un conocimiento del léxico, pero además, para determinar si un hablante de una segunda lengua domina la destreza oral, es necesario implicarlo en una actividad en la que ponga directamente en práctica su lengua hablada (Weir, 2005).

Todos estos factores que definen la competencia comunicativa desde un punto de vista lingüístico y pragmático nos llevan a pensar que la responsabilidad de los evaluadores es grande y éticamente indispensable. Alarcón $(2007,191)$, habla de tres condiciones que deben de estar presentes en cualquier fase de evaluación: fiabilidad, validez y viabilidad. Estas tres condiciones deben tenerlas en cuenta tanto los sujetos evaluadores como los evaluados y de hecho así lo establece el MCERL (Marco Común Europeo de Referencia para las Lenguas. Consejo de Europa, 2001). Según Piñeiro (2007), la validez es el grado en que una prueba mide realmente lo que se pretende medir. Por ejemplo, en una prueba de expresión oral, lógicamente se pretende medir el grado de dominio del estudiante en esta destreza y no debería interferir ningún otro elemento como, por ejemplo, la habilidad epistemológica para interpretar un gráfico. La fiabilidad estudia hasta qué punto los resultados de la prueba son consistentes: si los candidatos hicieran el examen mañana después de haberlo hecho hoy, ¿obtendrían las mismas notas?" (Alderson, Clapham y Wall, 1995). La viabilidad está en relación con el ámbito de aplicación de la prueba y las circunstancias en las que se aplica. No es posible aplicar todas las pruebas en todas las situaciones de enseñanza. Depende, por ejemplo, de los recursos físicos, humanos y tecnológicos de los que se disponga (tiempo para hacer la prueba, profesorado para administrarla y corregirla, disponibilidad de medios audiovisuales, etc.).

Una de las asociaciones más reconocidas en la evaluación de segundas lenguas como es ALTE (Association of Language Testers in Europe, www.alte.org), en su 
código de buenas prácticas: Principles of Good Practice for ALTE Examinations (2001), expone cuatro puntos clave a la hora de evaluar con calidad: validity (validez), reliability (fiabilidad), impact (impacto) y practicability (viabilidad). El impacto, en este caso, se refiere a la imparcialidad de la evaluación que se realiza a la persona evaluada. Si tenemos en cuenta, como hemos dicho anteriormente, que uno de los problemas de la evaluación oral es la subjetividad, el impacto se convierte en un factor clave para evitarla.

Underhill $(1987,3)$, refiriéndose a la evaluación de la competencia oral, hace una distinción entre speaking proficiency, refiriéndose al aspecto comunicativo de la lengua y language proficiency, al aspecto más organizativo. Si analizamos esta distinción, es reminiscente de la postulada por Saussure en el año 1916 entre lengua y habla. Según Saussure, la lengua es el sistema utilizado como medio para la comunicación. Está en el subconsciente de los individuos y compuesta por signos. El habla es la realización del acto comunicativo utilizando la lengua. Es el acto voluntario y social del sujeto hablante y depende del contexto en el que se encuentre (Saussure, 1969). Desde nuestro punto de vista y de muchos expertos, el éxito comunicativo en una segunda lengua está en la comunicación efectiva, y no en la corrección formal de la lengua (Boonkit, 2010; Graddol, 2006). Esa comunicación efectiva se puede conseguir con una utilización adecuada de la lengua sin implicar su uso perfecto y sin errores.

Esta práctica hablada que es evaluada con un fin, ocurre en una alta proporción en el inglés del mundo profesional. Aunque esta evaluación implique un conocimiento de la lengua por parte de personal no lingüista e implique una inversión de tiempo, se hace necesaria en algunas situaciones dentro de una empresa. Esas situaciones pueden ser: selección de candidatos a un puesto de trabajo en una empresa dada, candidatos a una promoción interna y empleados de una empresa cuyo cometido requiere comunicarse habitualmente en inglés. El caso más problemático pero a su vez más importante es el primero: candidatos a un puesto de trabajo, ya que es el que requiere una solución más inmediata. Una vez que entra a formar parte de la empresa un individuo, es más costoso solucionar posibles problemas de competencia lingüística. De ahí que sea necesario llevar a cabo una correcta evaluación de competencia oral en la entrevista de trabajo y conocer la importancia del inglés profesional (Ongallo, 2007).

\subsection{El inglés en el mundo profesional}

No tendría sentido que no habláramos del inglés, cuando somos conscientes de que la comunicación oral es indispensable en el mundo de la empresa, no sólo como una segunda lengua, sino como lengua materna también, con el fin de alcanzar objetivos comerciales (Bargiela-Chiappini y otros, 2007).

Ellis y Johnson $(1994,37)$ simplifican la caracterización del discurso oral del inglés empresarial señalando que su principal característica es la actuación comunicativa o lo que ellos llaman communicative performance o actuación 
comunicativa en contraposición a la linguistic competence o competencia comunicativa en sentido estricto.

Ellis y Johnson $(1994,89)$, siempre dentro del mismo contexto comunicativo, recomiendan la evaluación realizando un análisis de discurso de inglés oral empresarial haciendo énfasis en las habilidades comunicativas, las funciones del lenguaje, la gramática y el léxico, contextualizándolo en situaciones reales o, en su defecto, en simulaciones de éstas. De hecho, la clasificación que hacen ellos mismos, se basa en dos tipos de comunicación: individual e interactiva. Implícito en la comunicación individual se encuentra el objetivo de facilitar comunicación a través de mensajes o presentaciones e incluso la comunicación existente en una entrevista de trabajo en la que se trata de demostrar el nivel lingüístico del candidato. En este caso, el candidato va a ser evaluado por personal no lingüista y de manera rápida y eficiente, algo que no siempre ocurre en la vida real profesional. De ahí que el final de este artículo se base en una propuesta de escala holística que facilite dicha labor en entrevistas de trabajo. Sin embargo, en la interacción nos encontramos con ejemplos de situaciones como las reuniones, formales y semiformales, las conversaciones telefónicas y la comunicación social. En estas situaciones profesionales, la comunicación depende no solo del nivel lingüístico sino de otras capacidades comunicativas sociolingüísticas y pragmáticas que hemos indicado anteriormente en nuestro marco teórico.

Cuando hablamos de lenguaje oral en el mundo profesional, no podemos olvidar un tipo de lenguaje complementario al de las palabras formado por los gestos, las posturas, las miradas que expresan estados de ánimo, consciente o inconscientemente, para expresar estados de ánimo o sentimientos: la comunicación no verbal. Su importancia es tal que se considera que, en una comunicación cara a cara, hasta el $65 \%$ de la información puede ser no verbal (gestos, movimientos, señales, etc.) (Ongallo, 2007). La comunicación no verbal y la verbal van unidas, ya que suelen emplearse juntas y de forma no contradictoria. En muchas ocasiones actúa como reguladora del proceso de comunicación, contribuyendo a ampliar o reducir la intensidad del mensaje o a matizar sus significados (Reinsch, 1996). El uso de esos sistemas de comunicación no verbal, es decir, los gestos, las manos, las posturas, las miradas, el tono o la velocidad al hablar también deberían de ser incluidos en las escalas de valoración que sean utilizadas por el evaluador, utilizando descriptores claros (Gibson, 2005).

Aun teniendo en cuenta todos estos factores para evaluar la competencia oral en el mundo profesional o fuera de éste, hay que seguir un proceso de evaluación determinado y detallado, el cual analizaremos en el siguiente apartado.

\subsection{El proceso de evaluación de la competencia oral}

La evaluación de la destreza oral en una segunda lengua es un proceso en el que cabe distinguir varias fases o etapas desde que existe una necesidad de evaluación hasta el momento en el que se realiza. Dichas fases, según Luoma (2004), son las 
siguientes: definición de la necesidad de una evaluación, planificación y realización de la tarea y evaluación de la tarea propiamente dicha. En cada fase pueden intervenir diferentes sujetos. En la primera fase, la definición de la necesidad de una evaluación, es el sujeto evaluado el que necesita que su destreza se valore con un fin o el sujeto evaluador sea quien necesita medirla con fines prácticos, como la contratación de un empleado competente en una segunda lengua. En la segunda fase, la planificación de la tarea, ya no sólo son sujetos, sino entornos físicos o instituciones las que entran en consideración. En la tercera fase, la realización de la tarea, sólo interviene el sujeto o los sujetos examinados y de forma habitualmente pasiva, el evaluador. En la última fase, la evaluación, son los evaluadores (y en ocasiones, las instituciones a las que pertenecen), los que intervienen. Todo este proceso se puede ver reflejado con mayor claridad en el siguiente gráfico, que resume el proceso de evaluación, según Luoma (2004).

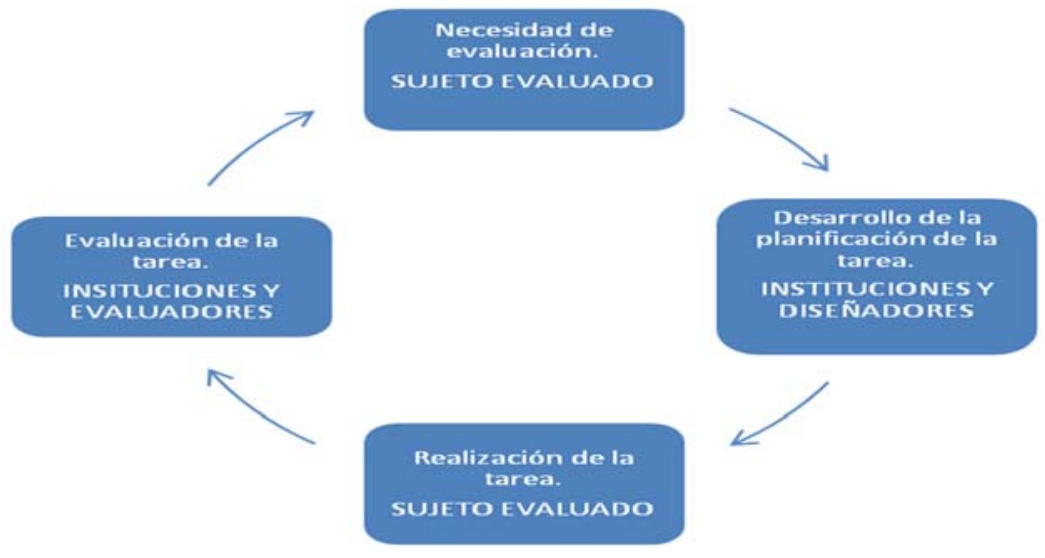

Fig. 1. Resumen del proceso de evaluación de Luoma (2004)

Veamos con detalle las fases del proceso, incluyendo un ejemplo práctico en el mundo laboral:

a) Necesidad de evaluación. La actividad del proceso comienza en la parte alta del gráfico cuando surge una necesidad de evaluación de la destreza oral que debe definirse. Citamos como ejemplo la selección de empleados que tengan un nivel de usuario independiente en una segunda lengua para promocionarlos al departamento de exportación e importación de una empresa. Previamente tienen que rellenar un cuestionario o test para conocer la capacidad inicial de competencia lingüística $y$ el interés en ser promocionado. 
b) Desarrollo y planificación de la tarea. En esta fase, los diseñadores de la tarea deben tener en cuenta principalmente las necesidades que tienen que analizar, para poder diseñar la tarea, establecer unos criterios de corrección y los procedimientos de administración de la prueba. En esta fase del proceso, se establece el tipo de evaluación que se va a realizar. Es decir, si va a evaluarse de forma cuantitativa con una nota numérica, por medio de una rúbrica o escala de valores, en la que se especifique si se cumplen objetivos o necesidades, o simplemente se asigna un nivel al individuo evaluado, tal como se hace en los exámenes de BULATS (Business Language Testing Services). ${ }^{1}$ de la Universidad de Cambridge. Un ejemplo de esta fase sería el de diseñar una prueba en la que los posibles candidatos anteriores tengan que enfrentarse a una situación comunicativa en inglés planteada y relacionada con el entorno de la exportación e importación y en la que tengan que proponer una solución con una extensión temporal de dos escasos minutos de discurso y grabado de forma online. La decisión de evaluación es la de evaluar la fluidez y el contenido comunicativo coherente del discurso.

c) Después de esta segunda fase del proceso de evaluación, continúa el ciclo por medio de dos de los procesos más importantes: el de realización de la tarea, que puede ser individual o de interacción y el de la evaluación o valoración de ésta. Siguiendo el ejemplo proporcionado de BULATS, los alumnos tienen un ordenador con micrófono y auriculares y tiempo asignado para realizar y grabar la prueba para que los evaluadores puedan llevar a cabo su tarea posterior.

d) La fase de evaluación, según Luoma, no es una última fase que cierre el proceso, sino que esa evaluación se transmite al evaluado con el fin de facilitar retroalimentación de los resultados de su producción, lo que demostrará que no es una acción mecánica (Luoma, 2004), sino que es una forma más de comunicación humana. Taylor y Galaczi (2011) también enfatizan el uso de la retroalimentación en el proceso de evaluación oral después de realizar la valoración. Según el ejemplo de proceso evaluador que hemos citado, después de realizar la evaluación, ésta tiene que ser comunicada a los participantes explicándoles las causas de la decisión que se ha tomado, la evaluación realizada y los errores cometidos.

Cabe señalar la existencia de otros métodos de evaluación. Así pues, Carroll (1980), sin embargo, sólo destaca tres fases en el proceso de evaluación oral: diseño de la prueba, desarrollo de la prueba y evaluación, omitiendo la fase inicial de las necesidades de dicha evaluación. Si trasladamos estas fases a las del ejemplo

${ }^{1}$ BULATS: Business Language Testing Services. Exámenes realizados por la Universidad de Cambridge para evaluar el nivel de inglés utilizado en el mundo profesional. 
presentado en el proceso de Luoma, Carroll no contaría ni con el análisis de necesidades de selección de los candidatos ni con la fase de retroalimentación de los resultados obtenidos, factores indispensables en cualquier proceso de evaluación.

En el proceso de evaluación oral, además, hay que tener en cuenta si se busca medir concretamente la producción y/o la interacción, ya que en cada una intervienen diferentes factores a tener en cuenta.

\subsection{La evaluación de la competencia oral en la producción individual y la interacción}

Charles (2007) diferencia dos tipos de comunicación oral: como proceso individual y como proceso recíproco. En la comunicación individual, normalmente el comunicador produce un monólogo que está condicionado por el tiempo y por una audiencia o contexto determinado, ya sea un monólogo, un discurso o una presentación. Sin embargo, cuando hay reciprocidad en la que existe un proceso de comunicación conjunta, hay que tener en cuenta otros aspectos adicionales como la comprensión mutua, la empatía, el uso de los turnos y la habilidad expresiva.

To test speaking ability we should require candidates to demonstrate their ability to use language in ways which are characteristic of interactive speech. Obviously, for certain contexts we are also interested in their ability to perform extended monologues where informational routines are likely to predominate. (Weir, 2005, 103)

En la evaluación de la producción oral individual, hay que tener en cuenta si ésta se produce de forma espontánea o el discurso ya ha sido preparado con anterioridad. En el primer caso, el uso del lenguaje es más natural e improvisado, por lo que cabe esperar la aparición y aceptación natural de ciertos rasgos (tartamudeos, falta de coordinación morfológica, etc.) que no serían admitidos en el discurso planificado. Según Hughes (2011), la producción oral espontánea está llena de pausas y dudas, incluso para los hablantes nativos. Aunque se pueda valorar más la capacidad expresiva de la lengua a tiempo real, el vocabulario será más limitado, la incorrección gramatical más frecuente y las repeticiones para llenar el discurso serán recursos comunes.

La interacción lingüística se desarrolla por medio de actuaciones individuales que interaccionan con otras. Por lo tanto, al haber una negociación cognitiva de significados y mensajes, la evaluación no se puede basar solamente en componentes aislados como pronunciación, gramática, fluidez y coherencia, por ejemplo, sino que se debería tener en cuenta el desarrollo bilateral o multilateral de la conversación, la reacción de los hablantes ante la reciprocidad discursiva y la intención de continuidad de la conversación (Douglas, 1997). Al igual que en el uso de la propia lengua, hay que tener en cuenta ciertos factores en dicha evaluación, como es el contexto de aplicación real, que debe ser apropiado desde el punto de 
vista de la aproximación a las situaciones reales prototípicas de uso (Hughes, 2011). Con respecto a este último punto, creemos que es fundamental que en la evaluación oral se simule un contexto parecido a la realidad a la que se puede enfrentar el evaluado. De esa manera, se logrará evaluar la competencia oral desde el punto de vista pragmático (Douglas, 1997).

Ante tales decisiones por parte de los evaluadores y con el fin de disminuir la subjetividad en el momento de evaluar oralmente, aumentando por lo tanto la validez de la tarea, se hace indispensable el uso de criterios evaluadores comunes y razonables en dicho proceso. Ese criterio debe ser válido tanto para un evaluador individual como para una comunidad de evaluadores que necesiten llegar conjuntamente a un consenso. De ahí que existan las escalas de valoración o de evaluación, como veremos en el siguiente apartado.

\section{LAS ESCALAS DE VALORACIÓN DE LA COMPETENCIA ORAL: PROPUESTA DE ESCALA HOLÍSTICA}

Las escalas de valoración nacen con el fin primordial de dar fiabilidad a la puntuación dada en una evaluación, especialmente cuando se hace necesario tomar una decisión o ejecutar una intervención (Taylor y Galaczi, 2011). North (2000) describe el reto de realizar escalas de valoración para producción oral como "trying to describe complex phenomena in small number of words on the basis of incomplete theory". Luoma (2004) está de acuerdo con esta observación ya que es consciente de la dificultad de resumir descriptores que faciliten su uso a la hora de evaluar a varios hablantes. Brindley $(1998,16)$ añade que no es fácil asignar los descriptores necesarios para diferenciar entre lo que el hablante debería decir y lo que dice realmente. De cualquier manera, las escalas de valoración constituyen una forma de evaluación útil, ya que permiten a los expertos valorar la actuación de un hablante y juzgar en qué grado es mejor o peor su producción según los objetivos marcados (Luoma, 2004). Shaw y Weir $(2007,143)$, además, justifican el uso de las escalas de valoración como un medio de acuerdo y unificación de criterios entre varios evaluadores.

Taylor y Galaczi (2011) conciben una relación triangular entre tres factores esenciales a incluir en una escala de valoración: la habilidad cognitiva del hablante, la tarea y contexto de la prueba a realizar y la evaluación en sí. Estos factores deben tenerse en cuenta para evitar que las escalas se conviertan en un mero proceso matemático. Para ambas autoras, las escalas de valoración son indispensables para cualquier evaluación oral y hay que invertir tiempo en incluir descriptores y escalas apropiados. Si no se presta la suficiente atención a este hecho, se corre el riesgo de incurrir en irrelevancia y falta de validez. Además, se valora la importancia de la coevaluación de varios profesionales con la misma escala de valoración para un mismo individuo con el fin de llegar a un consenso útil y válido. Alderson (1991) y North (1996/2000) aconsejan preparar diferentes versiones de las escalas dependiendo de los hablantes, en lugar de realizar una única escala que sea de 
utilidad para todos los hablantes en general. Veamos qué tipos de escala de valoración de segundas lenguas existen según los criterios de evaluación.

\subsection{Tipos de escalas de valoración}

Los tipos de escalas de valoración de evaluación oral se clasifican en dos categorías generales: holística y analítica. La evaluación holística evalúa la producción oral desde un punto de vista unitario y global, evaluando la calidad de la actuación sin hacer subdivisiones ulteriores. La evaluación analítica identifica diferentes características de la producción oral y las analiza de forma individual aportando más riqueza informativa a la evaluación (Taylor y Galaczi, 2011). En el siguiente apartado analizaremos cada tipo, mencionando las ventajas y desventajas de cada uno.

\section{a) Escalas holísticas}

Las escalas holísticas reflejan la impresión general o impression mark (ALTE, 1998, 147) del evaluador sobre una actuación de forma global. Al facilitar sólo una puntuación o valoración finales, resultan útiles para funcionalidades en las que hay que tomar una decisión como puede ser aprobar o suspender o aceptar o rechazar a un trabajador en un empleo (Luoma, 2004).

Una de las mayores ventajas de la evaluación holística estriba en la rapidez y simplicidad, especialmente en la evaluación masiva de individuos donde hay que facilitar un resultado, tomar una decisión, o asignar un nivel determinado. De la misma manera, se suele aplicar este tipo de evaluación cuando sólo se quiere evaluar una característica de la lengua o asignar un nivel determinado. Los descriptores de la evaluación holística ofrecen la posibilidad de realizar una evaluación descriptiva e incluso intuitiva y accesible para realizar una autoevaluación (Portfolio Europeo de las Lenguas ${ }^{2}$ o PEL) o evaluaciones informales por parte de hablantes no expertos. Podemos ver ejemplos de dos escalas holísticas posteriormente. Veamos como ejemplo la escala de valoración que se utiliza en los exámenes de BULATS ${ }^{3}$ en los que se asigna un nivel europeo, pero no una puntuación diferenciada por destrezas.

\footnotetext{
${ }^{2}$ El Portfolio Europeo de las Lenguas (http://www.oapee.es/oapee/inicio/iniciativas/ portfolio.html) es un documento de uso personal promovido por el Consejo de Europa, en el que los que aprenden o han aprendido una lengua - ya sea en un entorno formal o de aprendizaje - pueden registrar sus experiencias de aprendizaje de lenguas y culturas y reflexionar sobre ellas.

${ }^{3}$ BULATS (www.bulats.org/es), utiliza una evaluación de la lengua a escala internacional para la contratación en empresas y en el sector industrial, la identificación y provisión de capacitación, la admisión a cursos de negocios, la evaluación de la eficacia de los cursos de idiomas y la capacitación en este ámbito.
} 


\begin{tabular}{|c|c|}
\hline BAND & GLOBAL DESCRIPTORS \\
\hline 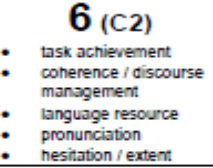 & $\begin{array}{l}\text { Fully operational command of the spoken language } \\
\text { - Achieves the task effectively; responses are consistently appropriate. } \\
\text { - Able to express both simple and complex ideas with ease; coherent extended discourse. } \\
\text { - Consistently, displays wide range and accurate use of grammar and vocabulary. } \\
\text { - Pronunciation is easy to understand; stress, thythm and intonation are used to express meaning effectively. } \\
\text { Responds promptly with only natural hesitation; makes effective use of the allowed response time. }\end{array}$ \\
\hline $\begin{array}{l}\quad 5 \text { (C1) } \\
\quad \text { task achievement } \\
\text { conerence / discourse } \\
\text { management } \\
\text { - language resource } \\
\text { - } \\
\text { pronunciation } \\
\text { hesitation / extent } \\
\end{array}$ & $\begin{array}{l}\text { Good operational command of the spoken language } \\
\text { - Achieves the task well; responses are generally appropriate. } \\
\text { - } \quad \text { Generally, displays wide range and accurate use of grammar and vocabulary. } \\
\text { - Pronunciation is easy to understand; stress, ihythm and intonation are used to express meaning well. } \\
\text { - Generaly responds prompty, with only natural hesitation; generally makes good use of the allowed response } \\
\text { time. }\end{array}$ \\
\hline 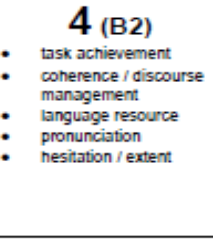 & $\begin{array}{l}\text { Generally effective command of the spoken language } \\
\text { - Achieves the task adequately; most responses are appropriate but a few may be inappropriate or ambiguous } \\
\text { (possibly due to incomprehension of input). } \\
\text { - Able to express simple ideas and makes some attempt to express complex ideas; mostly coherent, with some } \\
\text { extended discourse. } \\
\text { - There is an adequate range of grammar and vocabulary which is sulficiently accurate to deal with the tasks. } \\
\text { - Pronunciation can generally be understood; stress, rhythm and intonation are used to express meaning } \\
\text { adequately. } \\
\text { May be some hesitation while searching for language; generally makes adequate use of the allowed response } \\
\text { time. }\end{array}$ \\
\hline 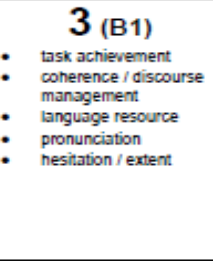 & $\begin{array}{l}\text { Limited but effective command of the spoken language } \\
\text { - Achieves most of the task, in a limited way; some responses may be inappropriate, ambiguous or not } \\
\text { attempted (possibly due to incomprehension of input). } \\
\text { - Able to express simple ideas; little extended discourse; some incoherence. } \\
\text { The range of grammar and vocabulary used is sufficient to complete tasks in a limited way. Some language in } \\
\text { simple utterances is accurate but basic inaccuracies may impede communication of ideas and achievement of } \\
\text { the tasks. } \\
\text { Pronunciation can generally be understood but L1 features may cause strain; an attempt is made to use } \\
\text { aspects of stress, thythm and intonation to express meaning. } \\
\text { Hesitation may demand patience of the listener, use of the allowed response time may not always be } \\
\text { adieguate. }\end{array}$ \\
\hline
\end{tabular}

Tabla. 1. Escala de valoración holística para competencia oral de BULATS

La escala de valoración holística, por otro lado, ofrece una desventaja a la hora de propiciar información evaluadora: no ofrece la posibilidad de acreditar o penalizar las fortalezas o debilidades de la producción oral $\mathrm{y}$, por lo tanto, es poco útil para la retroalimentación (Weir, 1994).

\section{b) Escalas analíticas}

Las escalas analíticas difieren de las holísticas en que la evaluación se hace de forma descompuesta por medio de características comunicativas que son analizadas individualmente. Este tipo de escala de valoración es útil en procesos diagnósticos de evaluación (Taylor y Galaczi, 2011). Las escalas analíticas suelen contener de tres a cinco criterios o categorías, las cuales tienen a su vez descriptores de diferentes niveles. Dichas escalas forman una cuadrícula en la cual se incluye la puntuación que determina las fortalezas y debilidades de los individuos examinados según la producción oral (en el caso que nos ocupa) (Luoma, 2004). Davies y otros (1999) señalan que las categorías incluidas en las escalas analíticas de evaluación oral suelen ser comunes, al menos parcialmente: pronunciación, comprensión, fluidez, corrección y coherencia. Veamos las categorías incluidas en las escalas de 
valoración oral de tres grandes instituciones evaluadoras: Consejo de Europa (2001: 28-29), Universidad de Cambridge, ESOL Examinations (2010) e International English Language Testing System (IELTS) (2009).

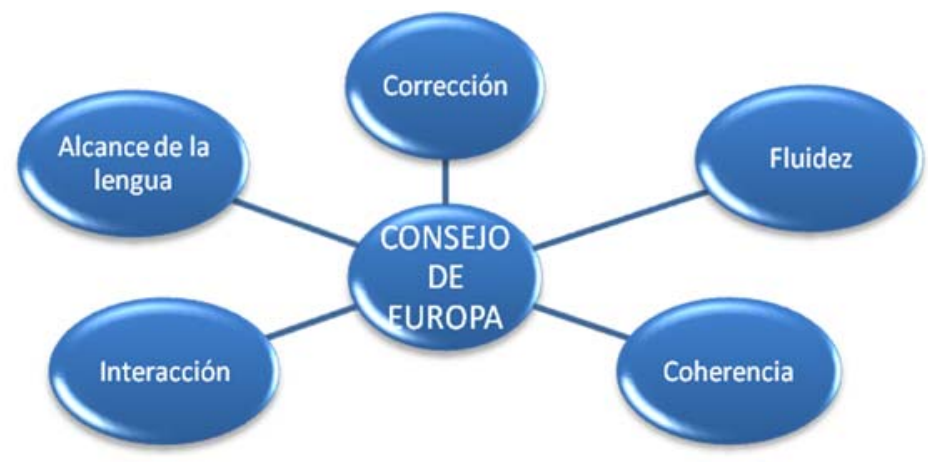

Fig. 3. Criterios de evaluación de inglés del Consejo de Europa

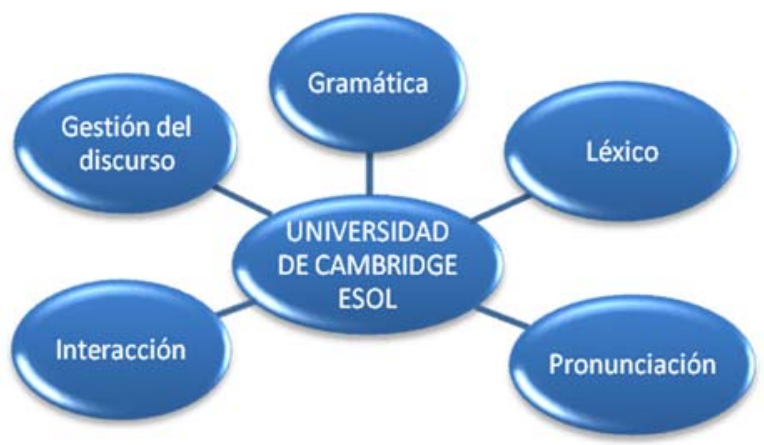

Fig.4. Criterios de evaluación de inglés de la Universidad de Cambridge

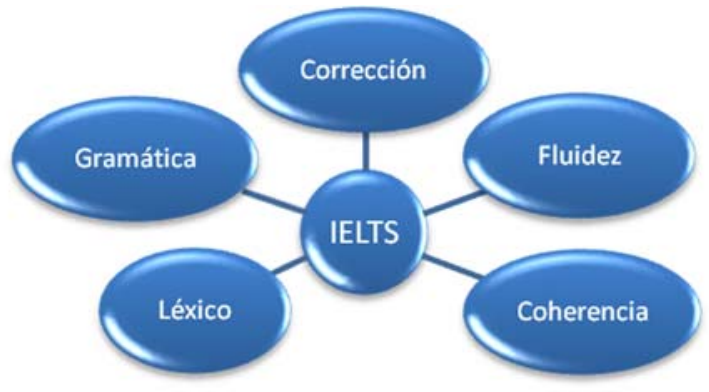

Fig. 5. Criterios de evaluación de inglés de IELTS (www.ielts.org) 
Podemos comprobar a través de estas figuras que algunas veces los criterios se repiten, aunque pertenezcan a instituciones examinadoras diferentes. Con respecto al número de categorías necesarias para crear una escala de valoración, en el MCERL (2001) aparecen catorce para incluir en las escalas de valoración de producción oral de interacción: estrategias de turnos de palabra, desarrollo temático, estrategias de cooperación, precisión, solicitud de aclaraciones, adecuación sociolingüística, fluidez, expresión oral en general, flexibilidad, riqueza de vocabulario, coherencia y cohesión, corrección gramatical, dominio de la pronunciación y dominio del vocabulario. Sin embargo, Luoma $(2004,80)$ señala que cinco categorías son suficientes para emitir una valoración sobre una acción comunicativa y que un número superior a siete ya supondría una sobrecarga psicológica para el evaluador. Si, además, el evaluador tiene que participar activamente en el acto comunicativo que se va a evaluar, haciendo el papel de interlocutor o rellenando una escala de valoración, la carga cognitiva es aún mayor (Taylor y Galaczi, 2011).

La gran ventaja de las escalas analíticas es que ayudan a analizar el juicio de los evaluadores de forma más estrecha o ajustada que las holísticas, contribuyendo a dar validez a la evaluación y a llegar a acuerdos entre ellos (Weir, 1990). Este hecho es fundamentalmente valioso en el proceso de aprendizaje de segundas lenguas, cuyo análisis puede ser de utilidad para el conocimiento detallado de habilidades y sub-habilidades comunicativas de los estudiantes, es decir, para la evaluación formativa.

Sin embargo, la escala analítica tiene también alguna otra desventaja, además de la sobrecarga cognitiva para el evaluador que hemos mencionado anteriormente. Una escala analítica con demasiadas categorías puede desviar la atención de la competencia comunicativa general que se va a evaluar hacia cuestiones específicas que empañen una visión de conjunto (Hughes 2003, 103-4). De hecho, esas subcategorías deberían ser agrupadas o combinadas con el fin de crear una valoración global (Taylor y Galaczi, 2011). Otra desventaja del uso de las escalas analíticas, según estas mismas autoras, es el consumo de tiempo tanto en preparar la escala como en evaluarla, lo que supone también una necesidad económica mayor al necesitar más evaluadores y más tiempo.

Como podemos ver, las tablas de valoración se ajustan a los objetivos y a las necesidades de las instituciones, por lo que sería interesante que cada centro, cada evaluador o institución pudiera adaptar en consenso una determinada escala a su necesidad evaluadora de una forma justa y equitativa. Como en nuestro caso, no estamos hablando de un centro sino del mundo profesional en general, no queremos terminar sin ofrecer una propuesta de evaluación ante una situación que requiere rapidez y evaluación holística como ocurre en los procesos de selección masiva de candidatos a una empresa en la que se requiere conocimientos de inglés. 


\subsection{Propuesta de escala holística}

Teniendo en cuenta las apreciaciones de Valcárcel y Verdú (1995), en las que dicen que el contenido de la comunicación no es suficiente para medir la capacidad lingüística comunicativa de un alumno, sino que habrá que especificar con qué precisión ese alumno es capaz de expresar tanto las nociones como las funciones y esto implica juicios de valor objetivos y que ese nivel de precisión, y teniendo en cuenta que, tal como especifica Luoma (2004), la escala de valoración depende del tipo de los fines evaluadores, se ha diseñado aquí una propuesta de escala de valoración.

Esta escala evaluará la comunicación basada en una tarea propuesta a los candidatos en el momento de la entrevista de selección, ya sea como comunicación individual como interacción. Según Luoma (2004), hay que tener en cuenta, además de la precisión, coherencia, alcance de la lengua y habilidades de interacción, la pronunciación y el lenguaje corporal. De ahí que, teniendo en cuenta que la escala de valoración de la competencia oral utilizada en el MCERL (alcance de la lengua, precisión, fluidez, y coherencia), no incluye todas las características indispensables para que exista una completa valoración de la competencia oral, desde nuestro punto de vista, se ha decidido añadir a la escala de valoración propuesta, dos nuevos descriptores extraídos de la escala de valoración de competencia oral utilizada en Vancouver Community College $e^{4}$ que, además de las características anteriores añaden en sus escalas de valoración: pronunciación y lenguaje corporal.

En la tabla siguiente, podemos ver el modelo de rúbrica seguida para la evaluación individual, junto con una pequeña tabla con descriptores simples y generales de lo que significa cada característica de la escala.

Esta segunda tabla se realizó con el fin de mantener coherencia en la evaluación (Taylor y Galaczi, 2011) y para uso común de varios posibles evaluadores.

\begin{tabular}{|l|l|l|l|l|l|l|}
\hline $\begin{array}{c}\text { Evaluación } \\
\text { de 1(min) a } \\
\mathbf{3}(\mathbf{m a x})\end{array}$ & $\begin{array}{c}\text { Alcance } \\
\text { de la } \\
\text { lengua }\end{array}$ & Precisión & Fluidez & Coherencia & Pronunc. & $\begin{array}{c}\text { Lenguaje } \\
\text { corporal }\end{array}$ \\
\hline $\begin{array}{l}\text { Candidato } \\
\mathbf{1}\end{array}$ & & & & & & \\
\hline
\end{tabular}

Tabla 2. Modelo de la escala de valoración de la prueba individual

\footnotetext{
${ }^{4}$ Vancouver Community College es una de las universidades públicas más prestigiosas de Canadá. Fue fundada en 1965 y centra su enseñanza en el alumno desde hace décadas. En ella estudian cada año 26.000 alumnos de los cuales el 8\% está compuesto por estudiantes internacionales.
} 


\begin{tabular}{|c|l|}
\hline DESCRIPTORES & $\mathbf{1}($ pobre)/2(bien)/3(muy bien) \\
\hline $\begin{array}{c}\text { Alcance de la } \\
\text { lengua en general }\end{array}$ & $\begin{array}{l}\text { Repertorio de palabras y frases con el fin de comunicar de forma } \\
\text { efectiva. }\end{array}$ \\
\hline Precisión & Uso correcto de la gramática en la comunicación. \\
\hline Fluidez & Expresión fluida sin pausas o dudas. \\
\hline Coherencia & Coherencia del discurso y uso de conectores. \\
\hline Pronunciación & Entonación apropiada y articulación correcta de sonidos ingleses. \\
\hline Lenguaje corporal & $\begin{array}{l}\text { Uso de lenguaje no verbal o lenguaje gestual para captar la } \\
\text { atención de la audiencia. }\end{array}$ \\
\hline
\end{tabular}

Tabla 3. Descriptores de la escala de valoración individual

Con respecto a la escala de valoración de la interacción, decidimos mantener la misma escala de la producción individual, ya que de esa manera se podía hacer un estudio contrastivo de la producción de un hablante en una conversación en la que tiene que alterar el guión de la producción en tiempo real, por lo que la preparación del output es mínima. En este caso y siguiendo la generalidad y simplicidad de una escala holística diseñada para otros evaluadores o para empresas en posibles procesos de selección, decidimos añadir sólo el descriptor de interacción. Ante la generalidad definitoria de dicho término, como explicaremos en breve, creemos que dicho descriptor es la clave para comprender su importancia en la evaluación. Al centramos en los dos modelos seguidos en el diseño de las escalas de valoración (MCERL (2001) y Vancouver Community College, 2010), se hace necesario comparar las características que definen la interacción en cada uno de los modelos, como veremos en las tablas siguientes.

\begin{tabular}{|l|l|}
\hline \multicolumn{1}{|c|}{ Interacción (MCERL) } & \multicolumn{1}{c|}{\begin{tabular}{c}
\multicolumn{1}{c|}{ Interacción (Vancouver Community } \\
College)
\end{tabular}} \\
\hline Turnos de palabra & Comenzar una conversación \\
\hline Colaboración & $\begin{array}{l}\text { Comprobar si el interlocutor ha } \\
\text { comprendido }\end{array}$ \\
\hline Desarrollo temático & Introducir nuevas ideas en la conversación \\
\hline Mantenimiento de la conversación & $\begin{array}{l}\text { Mantener la conversación } \\
\text { Escuchar y responder al interlocutor de } \\
\text { forma apropiada }\end{array}$ \\
\hline
\end{tabular}

Tabla 4. Características de la interacción según el MCERL y Vancouver Community College

Las definiciones de la interacción en ambos casos coinciden en el hecho de que la interacción es una conversación que hay que mantener y para eso hay que escuchar y responder de forma participativa y colaborativamente, es decir, 
facilitando la comprensión del interlocutor, colaborando y respetando el turno de palabra. De ahí que en la escala de interacción añadiéramos el descriptor de interacción definido de forma general de la siguiente manera:

\begin{tabular}{|l|l|}
\hline Interacción & $\begin{array}{l}\text { Mantener la conversación, participando, respetando el turno de palabra y } \\
\text { colaborando. }\end{array}$ \\
\hline
\end{tabular}

Hay que añadir que esta escala ha sido mostrada a varios departamentos de Recursos Humanos, considerándola apropiada para posibles intervenciones. Por eso consideramos que la evaluación a través de esta escala de valoración comparándola con una escala analítica u otro procedimiento de evaluación, puede considerarse como una línea de investigación futura.

\section{CONCLUSIONES}

Como hemos visto a lo largo de este artículo, la evaluación oral de una segunda lengua es de suma importancia en el proceso de aprendizaje y enseñanza. Se ha analizado la literatura sobre las características de la competencia comunicativa oral, su evaluación y la complejidad de ésta, no solo desde el punto de vista lingüístico, sino desde el punto de vista pragmático y puramente evaluador y justo, ya que este proceso puede implicar subjetividad que puede ser paliada con la elaboración de una escala de valoración elaborada y adaptada a las necesidades de cada institución y de sus objetivos. Hemos analizado el proceso de evaluación con las fases y los elementos que tienen que intervenir para que la evaluación se haga de forma correcta. En dicho proceso, hay que tener en cuenta al evaluado, no solo al evaluador, ya que una evaluación que no tiene una retroalimentación implícita, no suele aportar ningún aspecto formativo a esa evaluación, lo cual implica un aspecto muy importante en el aprendizaje de una segunda lengua que no siempre se tiene en cuenta. El sujeto evaluado también tiene que ser tenido en cuenta al analizar las necesidades de éste antes de la evaluación. Esa necesidad u objetivo de la evaluación es especialmente importante en el mundo profesional, en el que se realiza una evaluación siempre con algún objetivo de promoción, de objetivos de negocio, o de entrevistas de trabajo. Dentro de ese proceso evaluador, hay que distinguir las diferencias de evaluación entre la producción individual y la interacción, las cuales no se producen con la misma fluidez y los factores que influyen en esa evaluación son diferentes. La interacción, que goza de suma importancia en el MCERL, implica una actuación que depende de los conocimientos de los interlocutores pero que a su vez reflejan la simulación conversacional de la vida real que tan importante es en el mundo profesional.

Con el fin de que haya consenso y similitud en cuanto a criterios de evaluación, es indispensable realizar y seguir las escalas de valoración que contengan descriptores apropiados y adaptados a los objetivos de evaluación. Dependiendo de 
los fines evaluadores, se seguirán escalas holísticas o analíticas. Hemos visto que las analíticas son más elaboradas y permiten llegar a un acuerdo entre diferentes evaluadores y favorecer evaluaciones diagnósticas. Sin embargo, las escalas holísticas, las cuales se utilizan en situaciones en las que se requiere una evaluación rápida, ya sea para aprobar o suspender, para aceptar a un trabajador en una empresa, para ascender en un puesto de trabajo o para asignar un nivel, son las más necesarias en los entornos profesionales. Por eso hemos querido hacer una propuesta de escala de evaluación ante una situación común en el mundo empresarial y que los mismos empresarios encuentran compleja para evaluar ya que nos son lingüistas: la selección de candidatos en entrevistas de trabajo masivas. Para ello se ha creado una rúbrica holística con descriptores indispensables en la comunicación oral en una segunda lengua con un sistema de valoración simple y rápido con el fin de facilitar a las empresas la valoración y discriminación de candidatos.

Todo lo dicho en este artículo, nos hace reflexionar sobre nuestra capacidad evaluadora como docentes, ya que la amplia experiencia no implica una correcta evaluación de competencia oral ni un conocimiento exhaustivo de los correctos procesos evaluadores y sus elementos. Debemos de tener en cuenta que el no seguimiento de todas estas pautas puede implicar un perjuicio para los alumnos o para los trabajadores de una empresa y es de sabios dudar de nosotros mismos y poder ser capaces de rectificar o de crear sistemas flexibles de evaluación que se puedan adaptar a las necesidades de los sujetos finales o de los interesados en la evaluación.

\section{BIBLIOGRAFÍA}

ALARCóN, Clara (2007): "Evaluar la expresión oral: propuesta contra los viejos inconvenientes. In Las destrezas orales en la enseñanza del español L2-LE". XVII Congreso Internacional de la Asociación del Español como Lengua Extranjera (ASELE): Logroño. Universidad de La Rioja. 191-204.

Alderson, J.Charles, C. ClaPhAM y D.WALl (1995): Language test construction and evaluation. Cambridge: Cambridge University Press.

Alderson, J.Charles (1991): "Bands and Scores". En J.C. Alderson y B. North (Eds), Language testing in the 1990's. Londres: MacMillan. 71-86.

ALTE (2001): Principles of good practice for ALTE examinations. www.alte.org [Consulta el 15 de marzo de 2016].

BACHMAN, Lyle (1990): Fundamental considerations in language testing. Oxford: Oxford University Press.

BACHMAN, Lyle Y A. PALMER (1982): "The construct validation of some components of communicative proficiency". Tesol Quarterly, 16(4), 449-465.

Bargiela-Chiappini, Francesca, C. Nickerson, y B. Planken (2007): Business discourse. Basingstoke: Palgrave Macmillan. 
BOUNKIT, Kaponman (2010): "Enhancing the development of speaking skills for non-native speakers of English". Procedia-social and behavioral sciences, 2, 1305-1309.

BRINDLEY, Giles. (1998): "Describing language development? Rating scales and SLA". En L.F. Bachman y A.D. Cohen (Eds), Interfaces between second language acquisition and language testing research. Cambridge: Cambridge University Press. 112-140.

CANALE, Michael (1995): "De la competencia comunicativa a la pedagogía comunicativa del lenguaje". En Competencia comunicativa: documentos básicos en la enseñanza de lenguas extranjeras (pp. 63-82). Edelsa.

CARROLL, Brendan (1980): Testing communicative performance. An interim study. Oxford: Pergamon Press.

CHARLES, Mirjaliisa (2007): "Language matters in global communication". Journal of Business Communication, 44, 260-282.

DougLAS, Dan (1997): "Testing speaking ability in academic contexts: theoretical considerations". TOEFL Monograph Series. Princeton, NJ: Educational Testing Services.

ELLIS, Mark y C. JOHNSON (1994): Teaching business English. Oxford: Oxford University Press.

GARCÍA-DEBENC, Claudine (2010): "Evaluar lo oral”. Enunciación, 15(2), 103-13.

GIBSON, Robert (2005): Intercultural business communication. Oxford: Oxford University Press.

GRADDOL, David (2006): English next. Londres: British Council.

Hughes, Arthur (2003): Testing for language teachers. Cambridge: Cambridge University Press.

HUGHES, Rebecca (2011): Teaching and researching speaking. Reino Unido: Pearson Education.

HYMES, Dell (1972): "On communicative competence" In: J.B. Pride and J. Holmes (Eds) Sociolinguistics. Selected Readings. Harmondsworth: Penguin, pp. 269293.

LuOMA, Sari (2004): Assessing speaking. Cambridge: Cambridge University Press

MCER: INSTITUTO CERVANTES (Trad.): Marco común europeo de referencia para las lenguas: aprendizaje, enseñanza, evaluación. Madrid: MECD-Anaya, 2002. (COUNCIL OF EUROPE: Common European framework of reference for languages: learning, teaching, assessment. Strasbourg. Council of Europe, (2001).

MCNAMARA, Tim (1996): Measuring second language performance. Londres: Longman.

NORTH, Brian (2000): The development of a common framework scale of language proficiency $(\mathrm{PhD})$. Nueva York: Peter Lang. 
O’MAlley, Michael y L. PIERCE (1996): Authentic assessment for English language learners: practical approaches for teachers. Nueva York: Addison Wesley.

Ongallo, Carlos (2007): Manual de comunicación: Guía para gestionar el conocimiento, la información y las relaciones humanas en empresas y organizaciones. Madrid: Librería-Editorial Dykinson.

PIÑEIRO, Mercedes (2007): "La evaluación de las destrezas orales". En Las destrezas orales en la enseñanza del español L2-LE: XVII Congreso Internacional de la Asociación del Español como lengua extranjera (ASELE): Logroño 27-30 de septiembre de 2006. 251-264. Universidad de La Rioja.

REINSCH, Lamar (1996): "Business communication: present, past, and future". Management Communication Quarterly, 10(1), 27-49.

SAUSSURE, Ferdinand (1969): Curso de lingüística general. Buenos Aires: Losada.

SHAW, Stuart y C. J. WEIR (2007): "Examining Writing: Research and Practice in Assessing Second Language Writing". En Studies in Language Testing, 26. Cambridge: Cambridge University Press. 130-134.

TAYLOR, Linda y E. GALACZI (2011): "Scoring Validity". En Studies in Language Testing: Examining Speaking, 30. Cambridge: Cambridge University Press. 412420.

UNDERHILL, Nic (1987): Testing spoken language: a handbook of oral testing techniques. Cambridge: Cambridge University Press.

VALCÁRCEL, SOlEDAD Y J. Verdú (1995): Observación y evaluación de la enseñanza comunicativa de lenguas modernas. Madrid: Centro de publicaciones.

WEIR, Cyril (1990): Communicative language testing. Nueva York: Prentice Hall.

WeIR, Cyril (1998): Communicative language testing with special reference to English as a FL. Exeter: University of Exeter Press.

WeIR, Cyril (2005): Language testing and validation. Hampshire: Palgrave MacMillan.

WeIR, Cyril y J. ROBERTS (1994): Evaluation in ELT. Oxford: Blackwell.

WIDDOWSON, Henry (1978): Teaching language as communication. Oxford: Oxford University Press. 